\title{
Integrated multislice CT and Tc-99m Sestamibi SPECT-CT evaluation of solitary pulmonary nodules
}

\section{TC multistrato e SPECT-TC con Tc-99m Sestamibi: diagnostica integrata nella caratterizzazione del nodulo polmonare solitario}

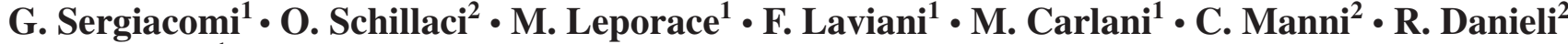 \\ G. Simonetti ${ }^{1}$
}

\begin{abstract}
${ }^{1}$ Dipartimento di Diagnostica per Immagini e Radiologia Interventistica, Università degli Studi di Roma "Tor Vergata", Roma, Italy ${ }^{2}$ Sezione di Medicina Nucleare, Università degli Studi di Roma "Tor Vergata”, Roma, Italy

Correspondence to: G. Sergiacomi, Policlinico "Tor Vergata", Viale Oxford 81, I-00133 Roma, Italy, Tel.: +39-06-20902372,

Fax +39-06-20902404, e-mail: sergiacomigianluigi@tin.it
\end{abstract}

Received: 22 March 2005 / Accepted: 23 August 2005 / Published online: 3 March 2006

\begin{abstract}
Purpose. The purpose of this study was to evaluate efficacy of multislice computed tomography (MSCT) and single photon emission computed tomography (SPECT)-CT with Tc-99m Sestamibi in the assessment of solitary pulmonary nodules of uncertain significance. Scintigraphy was performed using a 'hybrid' g-camera that allows simultaneous acquisition of SPECT and CT images, with interesting results in diagnostic oncology. Materials and methods. Between September 2003 and August 2004, 23 patients with a solitary pulmonary nodule detected on CT underwent SPECT-CT using Tc-99m Sestamibi as a radiotracer. Nodules with positive scintigraphy were immediately subjected to biopsy or surgical resection. Nodules with negative scintigraphy were followed up after 3-4 months by MSCT with automatic segmentation software (Advanced Lung Analysis, ALA) and histological characterisation.

Results. Of the 23 nodules (size range $0.8-2 \mathrm{~cm}$ ) discovered with MSCT, 11 showed intense uptake of Tc-99m Sestamibi. Ten lesions were true positive: seven adenocarcinomas, one squamous cell carcinoma, one large cell carcinoma and one metastasis. The only false positive was histologically classified as a large cell granuloma. Twelve lesions had negative scintigraphy: five fibrous lesions, three hamartomas, three granulomas and one adenocarcinoma (false negative). Benign nodules without tracer uptake underwent another CT scan 3-4 months later, which confirmed stability of the nodule size. Correlation of Sestamibi SPECT with histology showed sensitivity (Se) of $90.9 \%$, specificity (Sp) of $91.6 \%$, diagnostic accuracy of $91.3 \%$, positive predictive value (PPV) of $90.9 \%$ and negative predictive value (NPV) of $91.6 \%$.

Conclusions. The integrated use of MSCT and Tc-99m Sestamibi SPECT-CT could be very useful in the management of solitary pulmonary nodules (SPNs). In particular, in our preliminary study, scintigraphy provided significant diagnostic information to differentiate benign from suspicious pulmonary nodules. The use of scintigraphy could be helpful to anticipate histological assessment and surgical treatment of SPNs identified at CT.
\end{abstract}

Key words Multislice CT • Pulmonary nodule • Sestamibi • SPECT

\section{Riassunto}

Obiettivo. Scopo del nostro studio è valutare l'efficacia diagnostica dell'utilizzo della tomografia computerizzata multistrato (TCms) e della SPECT-TC con Tc-99m Sestamibi nella gestione di lesioni nodulari polmonari di incerto significato. La scintigrafia viene effettuata impiegando una g-camera "ibrida" che permette di acquisire immagini SPECT e TC nella stessa seduta d'esame, con risultati interessanti nella diagnostica oncologica.

Materiali e metodi. Ventitre pazienti, nel periodo tra settembre 2003 e agosto 2004, con un nodulo polmonare solitario sospetto, hanno eseguito una TCms del torace e successiva SPECT-TC utilizzando come radiotracciante il Tc-99m Sestamibi. I noduli positivi alla scintigrafia sono stati sottoposti immediatamente a tipizzazione istologica (biopsia o resezione chirurgica). I pazienti con noduli non captanti hanno eseguito un nuovo controllo TC a distanza di 3-4 mesi con complementare rielaborazione volumetrica mediante software dedicato Advantage Lung Analysis (ALA) e successiva caratterizzazione istologica.

Risultati. Dei 23 noduli (dimensioni 0,8-2 cm) individuati alla TCms, 11 sono risultati positivi all' esame scintigrafico. I veri positivi sono 10: 7 adenocarcinomi, un carcinoma squamocellulare, un carcinoma a grandi cellule ed una metastasi. L'unico falso positivo è stato tipizzato istologicamente come granuloma gigantocellulare. Le lesioni non captanti sono risultate 12: 5 esiti fibrosi, 3 amartomi, 3 granulomi ed un adenocarcinoma (falso negativo). Le nodularità di natura benigna non captanti sono rimaste volumetricamente stabili al successivo controllo TC a 3-4 mesi. L'esame scintigrafico con Sestamibi confrontato con il dato istologico ha riportato una sensibilità (Se) del 90,9\%, una specificità (Sp) del 91,6\%, un'accuratezza diagnostica del $91,3 \%$, un valore predittivo positivo (VPP) del 90,9\% ed un valore predittivo negativo (VPN) pari a 91,6\%. Conclusioni. L'utilizzo integrato della TCms e della SPECT-TC con Tc-99m Sestamibi può essere molto utile nella gestione del NPS. In particolare, nella nostra casistica preliminare, l'imaging scintigrafico ha fornito significative informazioni diagnostiche per differenziare le lesioni nodulari polmonari sospette. Ciò potrebbe permettere di anticipare la tipizzazione istologica e l'eventuale trattamento chirurgico in pazienti con NPS riscontrato all'esame TC del torace.

Parole chiave TC multistrato $\bullet$ Nodulo polmonare $\bullet$ Sestamibi $\bullet$ SPECT 


\section{Introduction}

Diagnostic imaging has achieved important results in the evaluation of solitary pulmonary nodules (SPNs) with multislice computed tomography (MSCT), thanks to major technological advances. This technique can now perform thinslice imaging of the lung parenchyma, and it employs software providing information on size, volume, density and contrast enhancement of pulmonary nodules as well as calculating automatically and accurately any change in size by comparing studies conducted at different times.

A few nodules can be defined as probably benign (presence of fat or benign calcification) and not requiring further investigation; others are highly suspicious for malignancy (spiculated margins, malignant calcification, diameter $>2 \mathrm{~cm}$ ) and require immediate surgical excision or biopsy [1]. The majority of pulmonary nodules (70\%), however, cannot be characterised with certainty, even after a CT scan.

Encouraging results in lung cancer diagnosis have been obtained with single photon emission computed tomography (SPECT) using 99mTc-hexakis-2-methoxy-isobutyl-isonitrile (Sestamibi), a lipophilic cation used in myocardial perfusion scintigraphy [2-4].

This technique currently makes use of new devices equipped with an X-ray tube-detector system for CT scans. Scintigraphy is carried out using a 'hybrid' g-camera allowing acquisition of SPECT and CT images during the same session. Preliminary results of the clinical application of this new diagnostic tool in the study of different neoplasms, including thoracic applications, have been very interesting $[5,6]$. This allows for a more effective and accurate preclinical diagnosis of disease, given that the early diagnosis of lung cancer is still the only means to improve patient survival $[7,8]$.

Our study investigated the characterisation of solitary nodules of uncertain significance discovered at chest radiography or incidentally during $\mathrm{CT}$ by integrating scintigraphy with MSCT. Our aim was to correlate the results of MSCT and Sestamibi SPECT-CT in the early diagnosis of lung cancer and to assess their concordance with histological examination.

\section{Materials and methods}

Between September 2003 and August 2004, we selected 23 patients (17 men and six women) aged between 40 and 82 years (mean age 63.2) with an SPN ranging from $0.8 \mathrm{~cm}$ to 2 $\mathrm{cm}$ in size (mean size $1.3 \mathrm{~cm}$ ) discovered at CT performed to investigate a chest radiography finding or as a diagnostic examination for another condition.

Our study excluded nodules with tomodensitometric characteristics highly suggestive of either benign or malignant disease. The examinations were conducted with a LightSpeed 16 MSCT scanner (GE Medical Systems, Milwaukee, WI, USA) capable of obtaining slice thicknesses between $10 \mathrm{~mm}$ and $0.625 \mathrm{~mm}$. The examination was performed dur-

\section{Introduzione}

La diagnostica per immagini ha raggiunto, grazie agli imponenti progressi tecnologici, importanti risultati nella valutazione del nodulo polmonare solitario (NPS) con l'ausilio della tomografia computerizzata multistrato (TCms). Attualmente tale tecnica è in grado di poter eseguire esami sul parenchima polmonare a strato sottile e si avvale di software in grado di fornire informazioni su dimensioni, volume, densità $e$ "contrast enhancement" delle nodularità polmonari nonché di calcolarne automaticamente in modo accurato le modificazioni dimensionali, comparando esami eseguiti in tempi diversi. Alcuni noduli possono essere definiti come benigni con alta probabilità (presenza di grasso o calcificazioni benigne) e non richiedere ulteriori approfondimenti; altri risultano altamente sospetti per malignità (margini spiculati, calcificazioni maligne, diametro superiore a $2 \mathrm{~cm}$ ) e richiedono immediata escissione chirurgica o biopsia [1]. Ciononostante la maggior parte dei noduli polmonari (70\%) non sono caratterizzabili con certezza anche dopo un esame TC.

Incoraggianti risultati nella diagnostica della neoplasia polmonare sono stati ottenuti con la tomografia computerizzata ad emissione di singolo fotone (SPECT) con l'utilizzo di Tc-99m-hexakis-2-metossi-isobutil-isonitrile (Sestamibi), un catione lipofilico in uso nella diagnostica scintigrafica miocardica perfusionale [2-4]. Tale tecnica si avvale ad oggi di nuove apparecchiature equipaggiate con un complesso tubo radiogeno-detettori per l'esecuzione di esami TC. La scintigrafia viene effettuata impiegando una g-camera "ibrida" che permette di acquisire immagini SPECT e TC nella stessa seduta d'esame. I risultati preliminari dell'impiego clinico di questa nuova apparecchiatura diagnostica nello studio di diverse neoplasie, comprendendo alcune applicazioni anche a livello toracico, si sono dimostrati molto interessanti [5, 6]. Tutto ciò ci consente di fare una diagnosi pre-clinica di malattia molto più efficace e accurata, dato che la diagnosi precoce delle neoplasie del polmone rimane l'unico mezzo per aumentare la sopravvivenza dei pazienti affetti $[7,8]$.

Il nostro studio è incentrato sulla caratterizzazione di lesioni nodulari solitarie di incerto significato rinvenute all'esame radiografico del torace, o incidentalmente durante l'esecuzione di esami TC, integrando l'esame scintigrafico con le informazioni fornite dalla TCms. Lo scopo è correlare i risultati forniti dalla TCms e dalla SPECT-TC con Sestamibi nella diagnosi precoce del cancro polmonare e valutarne la concordanza con l'esame istologico.

\section{Materiali e metodi}

Nel periodo compreso tra settembre 2003 e agosto 2004 sono stati selezionati 23 Pazienti di cui 17 uomini e 6 donne di età compresa tra i 40 e 82 anni (età media 63,2 anni), con un NPS di dimensioni comprese fra 0,8 e $2 \mathrm{~cm}$ (dimensioni medie 1,3 cm), individuato mediante esame TC, eseguito come approfondimento diagnostico di una precedente radiografia del torace o come accertamento diagnostico per altra patologia. Sono state escluse dalla nostra casistica le nodularità 
ing a single breathhold before and after administration of nonionic iodinated contrast material $(80-100 \mathrm{ml}, 3-4 \mathrm{ml} / \mathrm{s}$ flow rate, 25-30 s delay after starting the administration). Informed consent was obtained from all patients.

Acquisition parameters were as follows: slice thickness 5 $\mathrm{mm}$ (back reconstruction up to $0.625 \mathrm{~mm}$ ), feed 11.25 $\mathrm{mm} /$ rotation, rotation time $0.5 \mathrm{~s}$, total acquisition time $12-15$ $\mathrm{s}, 120-140 \mathrm{kV}, 200-240 \mathrm{~mA}$. Images were processed on an Advantage W 4.1 workstation supplied by the same company using multiplanar reconstructions (MPR), volume rendering (VR) and automatic segmentation software (ALA), which provides an accurate assessment of size, shape and density once the operator has selected the region of interest and performed three-dimensional (3D) segmentation with removal of adjacent structures.

Volume and morphology data obtained at the first measurement were compared with those of the follow-up examinations. Risk assessment was not performed on 2D criteria, such as changes in lesion diameter, but on 3D criteria, so that when the volume doubles, the lesion diameter actually increases by only $26 \%$.

All patients then underwent (within 5 days) lung scintigraphy with positive indicator using a VG Millennium (GE Medical Systems) dual-head gamma camera equipped with an X-ray tube-detector system similar to a third-generation CT [9]. This 'hybrid' system has shown to improve the diagnostic accuracy of a large number of SPECT examinations [5].

Twenty minutes after the IV administration of about 740 MBq of Tc-99m Sestamibi, SPECT-CT of the chest was performed. A total of 120 planar images (one every $3^{\circ}$ ) lasting $25 \mathrm{~s}$ each were acquired on a rotation arc of $360^{\circ}$ using a $128 \times 128$ matrix. Immediately afterwards, without moving the patient from the gamma camera bed, a low-resolution chest CT was done, with a field of view of $40 \mathrm{~cm}$, exactly equal to that of the SPECT acquisition.

Parameters used in this CT are fixed and cannot be changed by the operator: slice thickness $1 \mathrm{~cm}$, acquisition time approximately $14 \mathrm{~s}$ per section, $140 \mathrm{kV}$ and $2.5 \mathrm{~mA}$. The SPECT and CT images were processed with a dedicated workstation (Integra, GE Medical Systems), enabling rapid and accurate fusion of the relevant sections of the functional (SPECT) and anatomical (CT) studies.

In particular, SPECT images were processed using the method of filtered backprojections. After prefiltering with a Metz filter, the SPECT images were reconstructed in a ramp filter in order to produce transaxial sections from which coronal and sagittal images were derived.

Patients with positive nodules at scintigraphy underwent invasive procedures for histological characterisation (CTguided or bronchoscopic biopsy, video-assisted thoracoscopic excision).

Patients with negative nodules at scintigraphy underwent repeat CT after 3-4 months with reassessment of volume by means of ALA software and subsequent histological characterization (CT-guided or bronchoscopic biopsy, video-assisted thoracoscopic excision).

In these cases, follow-up CT was always performed with the same acquisition parameters as the first examina- con caratteristiche tomodensitometriche altamente probanti per benignità o malignità. Gli esami sono stati condotti con una TC multistrato Light-Speed 16 (GE Medical Systems, Milwakee, WI, USA) in grado di ottenere spessori di strato variabili da $10 \mathrm{~mm}$ a 0,625 $\mathrm{mm}$. L'esame è stato effettuato in un'unica apnea prima e dopo somministrazione di mezzo di contrasto organo-iodato non ionico (80-100 ml, flusso di 3-4 ml/s, ritardo di 25-30 s dall'inizio della somministrazione) previo consenso informato del paziente, con i seguenti parametri tecnici di acquisizione: spessore di strato pari a $5 \mathrm{~mm}$ (retroricostruibile fino a 0,625 mm), velocità di avanzamento $11,25 \mathrm{~mm} /$ rotazione, tempo di rotazione di $0,5 \mathrm{~s}$, tempo complessivo di acquisizione pari a 12-15 s, 120-140 $\mathrm{kV}, 200-240 \mathrm{~mA}$. Le immagini sono state successivamente rielaborate su una "workstation" Advantage W 4.1 fornita dalla stessa ditta mediante ricostruzioni multiplanari (MPR), volumetriche (VR) e software di segmentazione automatica (ALA) che permette, selezionando la regione di interesse ed effettuando una segmentazione tridimensionale con eliminazione delle strutture adiacenti, di ottenere una corretta valutazione di dimensioni, forma e densitometria. I valori ottenuti con le prime rilevazioni sono stati confrontati con quelli dei follow-up successivi, utilizzando come elemento di comparazione il criterio volumetrico accanto a quello morfologico. L'accertamento del rischio non viene effettuato sulla base di elementi bidimensionali, come le modificazioni del diametro della lesione, ma si fonda su criteri tridimensionali, così quando il volume raddoppia, il suo diametro aumenta in realtà solo del $26 \%$.

Tutti i pazienti sono stati successivamente (entro 5 giorni) sottoposti a tomoscintigrafia polmonare con indicatore positivo, impiegando una gamma camera a doppia testata VG Millenium (GE Medical Systems, Milwakee, WI, USA) equipaggiata con un complesso tubo radiogeno-detettori analogo ad una TC di III generazione [9]. Questo sistema "ibrido" si è rivelato utile per aumentare l'accuratezza diagnostica di molti esami SPECT [5]. Venti minuti dopo la somministrazione e.v. di circa $740 \mathrm{MBq}$ di Tc-99m Sestamibi è stato eseguito l'esame SPECT-TC del torace. Per la SPECT sono state acquisite 120 immagini planari (una ogni $3^{\circ}$ ) della durata di 25 s ognuna su un arco di rotazione di $360^{\circ}$, utilizzando una matrice $128 \times 128$. Subito dopo, senza muovere il paziente dal lettino della gamma camera, è stata effettuata una TC a bassa risoluzione del torace, su un campo di vista di $40 \mathrm{~cm}$, esattamente uguale a quello acquisito dalla SPECT. I parametri utilizzati da questa TC sono "fissi" $e$ non modificabili dall'operatore: spessore di strato di $1 \mathrm{~cm}$, tempo di acquisizione pari a circa 14 s per sezione, $140 \mathrm{kV}$ e 2,5 mA. Le immagini SPECT e TC ottenute sono state elaborate in una workstation dedicata (Integra, GE Medical System) che permette in maniera rapida di ottenere una fusione precisa delle corrispondenti sezioni dei due studi, funzionale (SPECT) ed anatomico (TC). In particolare, la SPECT è stata elaborata utilizzando il metodo delle retroproiezioni filtrate. Dopo una pre-filtrazione con un filtro di Metz, le immagini SPECT sono state ricostruite in un filtro a rampa per produrre sezioni transassiali, dalle quali sono state poi ottenute le immagini coronali e sagittali. I pazienti portatori di noduli con positività all'esame tomoscintigrafico, sono stati 
Table 1 Radiological, scintigraphic and histopathological features of 23 patients with solitary pulmonary nodules (SPNs). CT computed tomography, SPECT single photon emission computed tomography

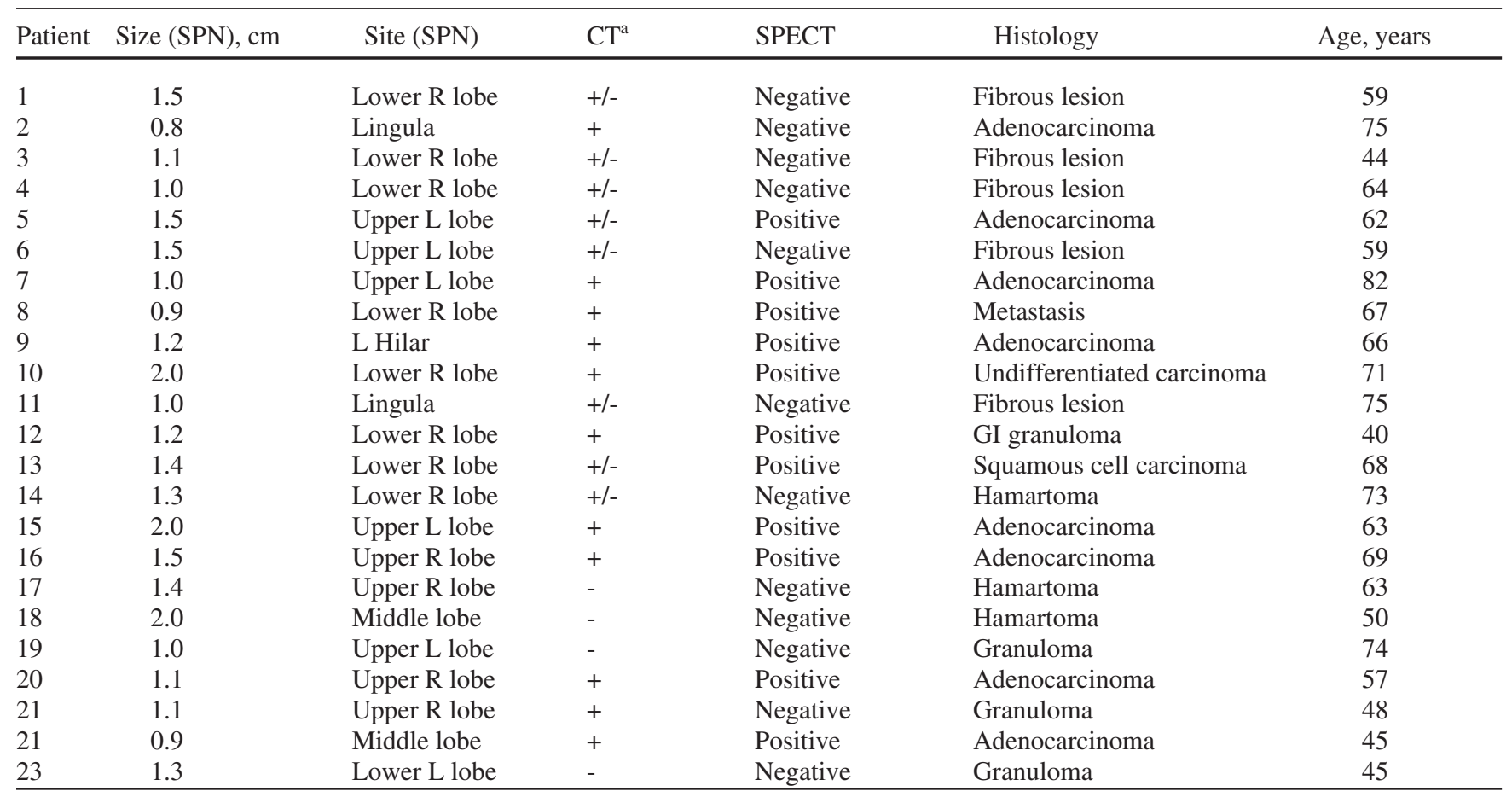

a + -, indeterminate; -, doubt about its benignity; +, doubt about its malignancy

$R$, right; $L$, left

Tabella 1 Caratteristiche riassuntive dei 23 pazienti con nodulo polmonare solitario: dato radiologico, scintigrafico ed istopatologico

\begin{tabular}{|c|c|c|c|c|c|c|}
\hline Paziente & $\begin{array}{l}\text { Dimensioni } \\
\text { (NPS), cm }\end{array}$ & Sede (NPS) & Aspetto $T C^{a}$ & SPECT & Istologia & Età, anni \\
\hline 1 & 1,5 & Lobo inf. $d x$ & $+/-$ & Negativa & Esito fibrotico & 59 \\
\hline 3 & 1,1 & Lobo inf. $d x$ & $+/-$ & Negativa & Esito fibrotico & 44 \\
\hline 4 & 1,0 & Lobo inf. $d x$ & $+/-$ & Negativa & Esito fibrotico & 64 \\
\hline 5 & 1,5 & Lobo sup. sn & $+/-$ & Positiva & Adenocarcinoma & 62 \\
\hline 8 & 0,9 & Lobo inf. $d x$ & + & Positiva & Metastasi & 67 \\
\hline 9 & 1,2 & Ilare sn & + & Positiva & Adenocarcinoma & 66 \\
\hline 10 & 2,0 & Lobo inf. $d x$ & + & Positiva & $K$ indiff. & 71 \\
\hline 11 & 1,0 & Lingula & $+/-$ & Negativa & Esito fibrotico & 75 \\
\hline 12 & 1,2 & Lobo inf. $d x$ & + & Positiva & Granuloma gig. & 40 \\
\hline 13 & 1,4 & Lobo inf. $d x$ & $+/-$ & Positiva & $K$ squamocellulare & 68 \\
\hline 17 & 1,4 & Lobo sup. $d x$ & - & Negativa & Amartoma & 63 \\
\hline 18 & 2,0 & Lobo medio & - & Negativa & Amartoma & 50 \\
\hline 19 & 1,0 & Lobo sup. sn & - & Negativa & Granuloma sp. & 74 \\
\hline 20 & 1,1 & Lobo sup. $d x$ & + & Positiva & Adenocarcinoma & 57 \\
\hline 21 & 1,1 & Lobo sup. $d x$ & + & Negativa & Granuloma & 48 \\
\hline 22 & 0,9 & Lobo medio & + & Positiva & Adenocarcinoma & 45 \\
\hline 23 & 1,3 & Lobo inf. sn & - & Negativa & Granuloma & 45 \\
\hline
\end{tabular}

${ }^{a}+/-$, indeterminato; -, dubbio sulla benignità; +, dubbio sulla malignità

$d x$, destro; sn, sinistro

tion $(\mathrm{kV}, \mathrm{mA}$, slice thickness, reconstruction interval, etc.) so as to ensure a reliable comparison. All patients, including those with negative lesions at scintigraphy reassessed sottoposti a procedure invasive per la tipizzazione istologica (biopsia TC guidata o broncoscopica, escissione videotoracoscopica). 
after 3-4 months, opted for definitive histological characterization rather than long-term follow-up.

\section{Results}

Of the 23 SPNs detected with CT, 11 were positive at scintigraphy and 12 were negative. Among the positive nodules, ten were found to be malignant by subsequent histological examination (size range: $0.8-2 \mathrm{~cm}$ ) (Fig. 1): seven adenocarcinomas, one squamous cell carcinoma, one undifferentiated large cell carcinoma and one myeloma metastasis. There was one false positive, which histology demonstrated to be a giant cell granuloma.

Of the 12 negative nodules (size range: $0.8-2 \mathrm{~cm}$ ), five were related to fibrosis, three were hamartomas (Figs. 2 and 3 ) and three were granulomas. There was one false negative that was found to be an adenocarcinoma. The smallest lesion $(0.8 \mathrm{~cm})$, this adenocarcinoma, measured $1 \mathrm{~cm}$ at CT followup after 3 months, with a significant increase in volume and therefore suspicious for malignancy.

Table 1 summarises the characteristics of the 23 patients in our study. In particular, the nodules examined were grouped based on well-known differential criteria used with CT. CT indicators of suspected benignity are well-defined contour, nonspiculated margins, fat tissue or calcification in more than $10 \%$ of the nodule and contrast enhancement $<20$ HU. CT indicators of suspected malignancy are blurred contour, spiculated margins, eccentric microcalcifications and contrast enhancement $>20 \mathrm{HU}$.

Nodules were considered "doubtful as to benignity or malignancy" when they showed a prevalence of no more than two criteria whereas they were considered "indeterminate" when they displayed no prevalence of any of the classification criteria.

On the basis of the correlation between the scintigraphic and histological results, the Sestamibi SPECT-CT scan had $90.9 \%$ sensitivity (Se), $91.6 \%$ specificity (Sp), diagnostic accuracy of $91.3 \%$ with positive predictive value (PPV) of $90.9 \%$ and negative predictive value (NPV) of $91.6 \%$ (Table 2).

In evaluating the scintigraphic scan, the simultaneous CT acquisition was always decisive in identifying nodular lesions with no uptake and contributed to the differential diagnosis in nodules with uptake located in perihepatic sites. SPECT acquisition alone without the aid of the anatomical CT images was decisive in all enhancing lung lesions other than those with perihepatic distribution. The CT scan with volumetric evaluation after 3-4 months showed absolute concordance with the histological findings (Table 3).

\section{Discussion}

Conventional chest radiography, the first-line diagnostic tool in all lung diseases, is not sufficiently sensitive or specific to ensure early diagnosis [10].
I pazienti portatori di noduli non captanti alla tomoscintigrafia sono stati sottoposti ad un nuovo controllo TC a distanza di 3-4 mesi con rivalutazione volumetrica mediante software ALA e successiva tipizzazione istologica (biopsia TC guidata o broncoscopica, escissione videotoracoscopica). In questi casi l'esame TC di controllo è stato sempre eseguito rispettando gli stessi parametri di acquisizione dell'esame precedente ( $k V, m A$, spessore di strato, intervallo di ricostruzione, ecc) al fine di ottenere una comparazione attendibile. Tutti i pazienti, anche quelli portatori di lesioni non captanti rivalutati a 3-4 mesi, hanno optato, previo consenso informato, per la definitiva caratterizzazione istologica piuttosto che sottoporsi ad ulteriori controlli per un lungo periodo di tempo.

\section{Risultati}

Dei 23 noduli polmonari solitari rinvenuti all'esame TC, 11 sono risultati positivi all'esame scintigrafico, 12 sono risultati negativi. Nei casi di noduli captanti la successiva valutazione istologica ha dimostrato la malignità della lesione in 10 casi (range dimensionale: 0,8-2 cm) (Fig. 1): 7 adenocarcinomi, un carcinoma squamocellulare, un carcinoma indifferenziato a grandi cellule ed una metastasi da mieloma. Un falso positivo si è dimostrato istologicamente un granuloma gigantocellulare. Dei 12 noduli non captanti (range dimensionale: 0,8-2 cm), 5 erano riferibili ad esiti fibrotici, 3 erano amartomi (Figg. 2 e 3), 3 granulomi ed infine uno è risultato falsamente negativo, in quanto si trattava di un adenocarcinoma. Quest'ultimo, risultata la lesione più piccola $(0,8 \mathrm{~cm})$ della nostra casistica, al successivo controllo TC a 3 mesi, presentava dimensioni pari a $1 \mathrm{~cm}$, con un incremento volumetrico significativo e pertanto sospetto per malignità. Le caratteristiche riassuntive dei 23 pazienti inclusi nel nostro studio sono riportate in Tabella 1. In particolare, le formazioni nodulari da noi esaminate sono state raggruppate in relazione a noti criteri differenziali utilizzati in tomo-

Table 2 Diagnostic accuracy of single photon emission computed tomography (SPECT)-CT

\begin{tabular}{lcc}
\hline & $\mathrm{n}$ & $\%$ \\
\hline Sensitivity (Se) & $10 / 11$ & 90.9 \\
Specificity (Sp) & $11 / 12$ & 91.6 \\
Positive predictive value (PPV) & $10 / 11$ & 90.9 \\
Negative predictive value (NPV) & $11 / 12$ & 91.6 \\
Accuracy & $21 / 23$ & 91.3 \\
\hline
\end{tabular}

Tabella 2 Accuratezza diagnostica della SPECT/TC

\begin{tabular}{lcc}
\hline & $n$ & $\%$ \\
\hline Sensibilità (Se) & $10 / 11$ & 90,9 \\
Specificità $(S p)$ & $11 / 12$ & 91,6 \\
Valore predittivo positivo (VPP) & $10 / 11$ & 90,9 \\
Valore predittivo negativo (VPN) & $11 / 12$ & 91,6 \\
Accuratezza & $21 / 23$ & 91,3 \\
\hline
\end{tabular}



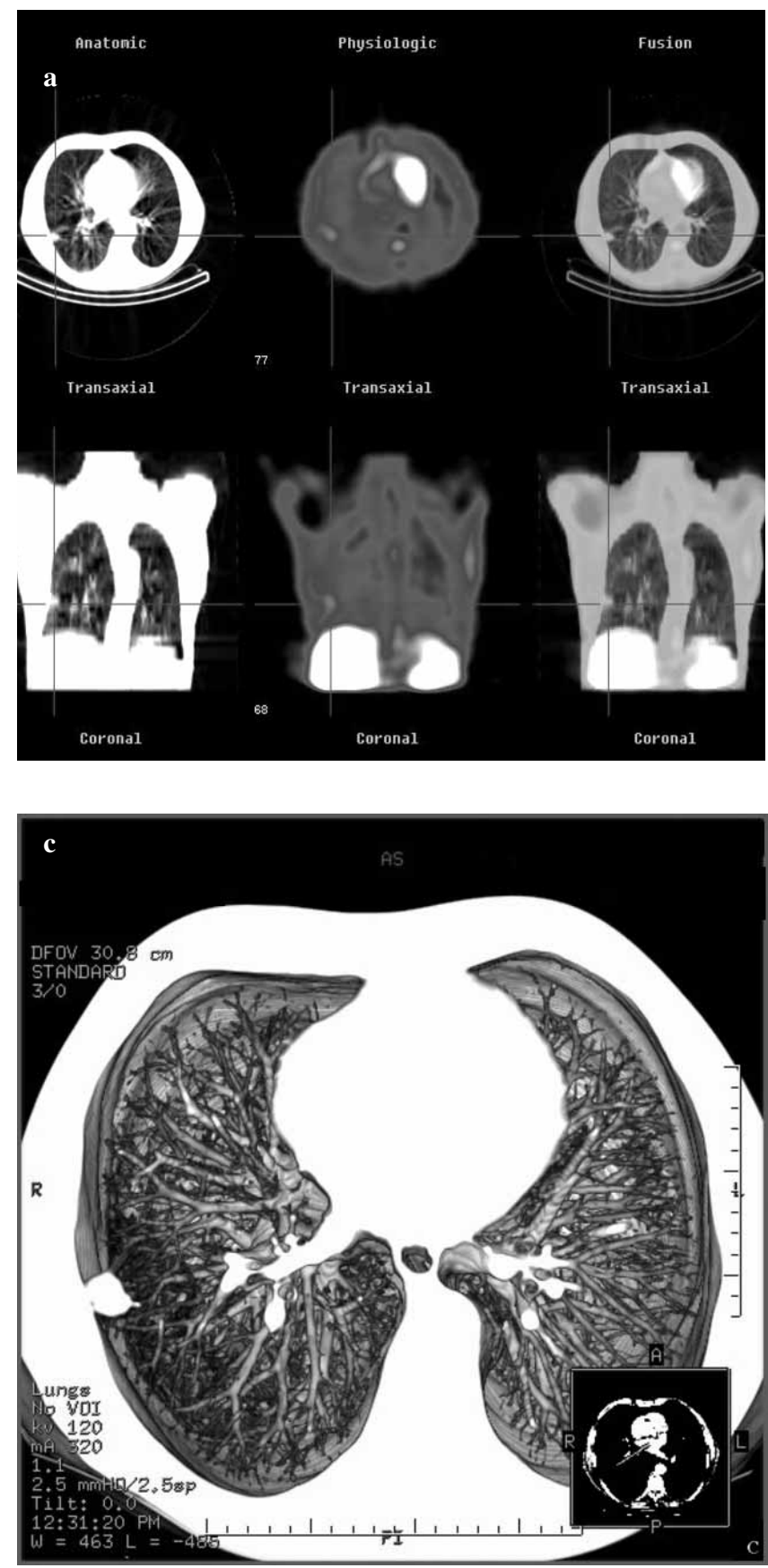

Diagnostic investigation based on standard chest radiography and sputum cytology failed to deliver the expected results in reducing mortality [11-13].

Over the last few years, the concept of early diagnosis of lung cancer has been reconsidered as a following the encouraging results obtained with multidetector computed tomography (MDCT) $[8,14,15]$ and the use complementary nuclear medicine methods. A solitary pulmonary nodule is defined as a rounded intraparenchymal opacity that is moderately well delimited, with a maximum diameter less than 3 $\mathrm{cm}$ [1]. CT differentiation of benign and malignant lesions is based on qualitative (nodule morphology) and quantitative (density, postcontrast enhancement characteristics, and le-

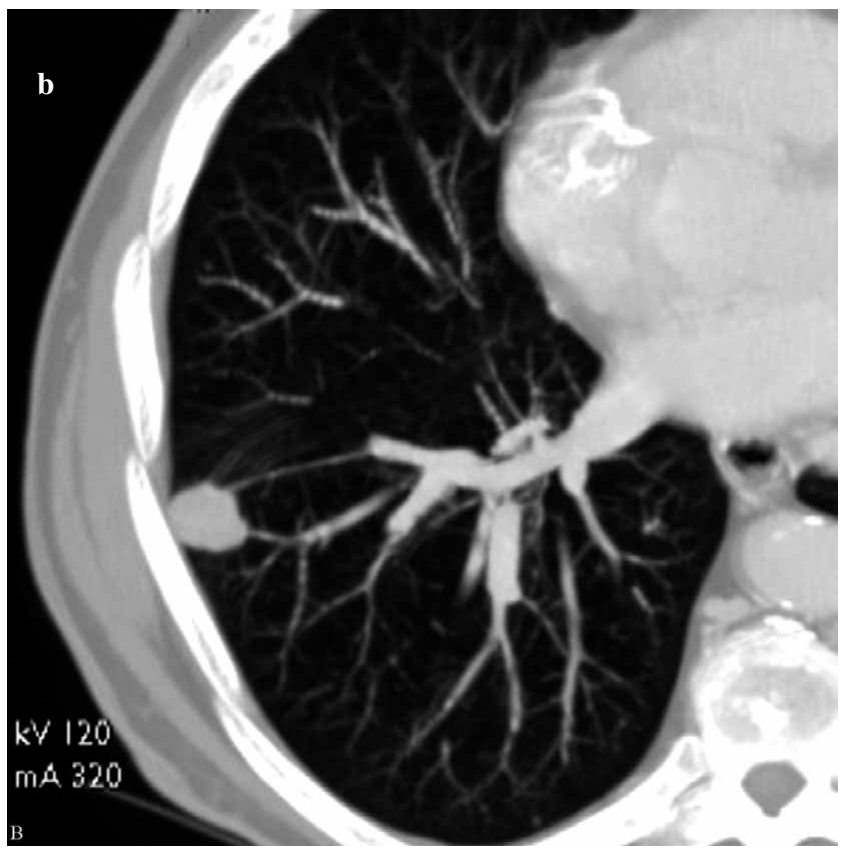

Fig. 1a Tc-99m Sestamibi single photon emission computed tomography (SPECT) lung scan of a 71-year-old man. The increased uptake of Sestamibi in the region of the nodule $(20 \mathrm{~mm})$ is clearly visible. b CT scan of the same patient showing a solitary pulmonary nodule in the right lower lobe. c Three-dimensional reconstruction. After surgical excision, the nodule was classified as malignant (anaplastic carcinoma).

Fig. 1a SPECT polmonare con Tc-99m Sestamibi in un uomo di 71 anni. È evidente una iperfissazione del radiofarmaco in corrispondenza del nodulo $(20 \mathrm{~mm})$. $\boldsymbol{b}$ TC dello stesso paziente che dimostra la presenza di una formazione nodulare localizzata nel lobo inferiore di destra. c Ricostruzione 3D. Dopo l'asportazione chirurgica il referto istologico ha confermato la natura maligna della lesione (carcinoma anaplastico).

grafia computerizzata. Indici TC di sospetta benignità sono la presenza di limiti ben definiti, margini non spiculati, presenza di tessuto adiposo o metaplasia calcifica in più del $10 \%$ del nodulo, un contrast enhancement $<20 \mathrm{UH}$. Indici $T C$ di sospetta malignità sono i limiti sfumati, i margini spiculati, le microcalcificazioni eccentriche, un contrast enhancement $>20$ UH. Abbiamo classificato le formazioni nodulari come "dubbie per benignità o malignità" quando presentavano una prevalenza di non oltre 2 criteri, mentre quelle definibili come "indeterminate" quando non era possibile evidenziare una prevalenza dei criteri classificativi utilizzati. L'esame SPECT-TC con Sestamibi, confrontando il dato scintigrafico col reperto istologico, ha riportato una sensibi- 

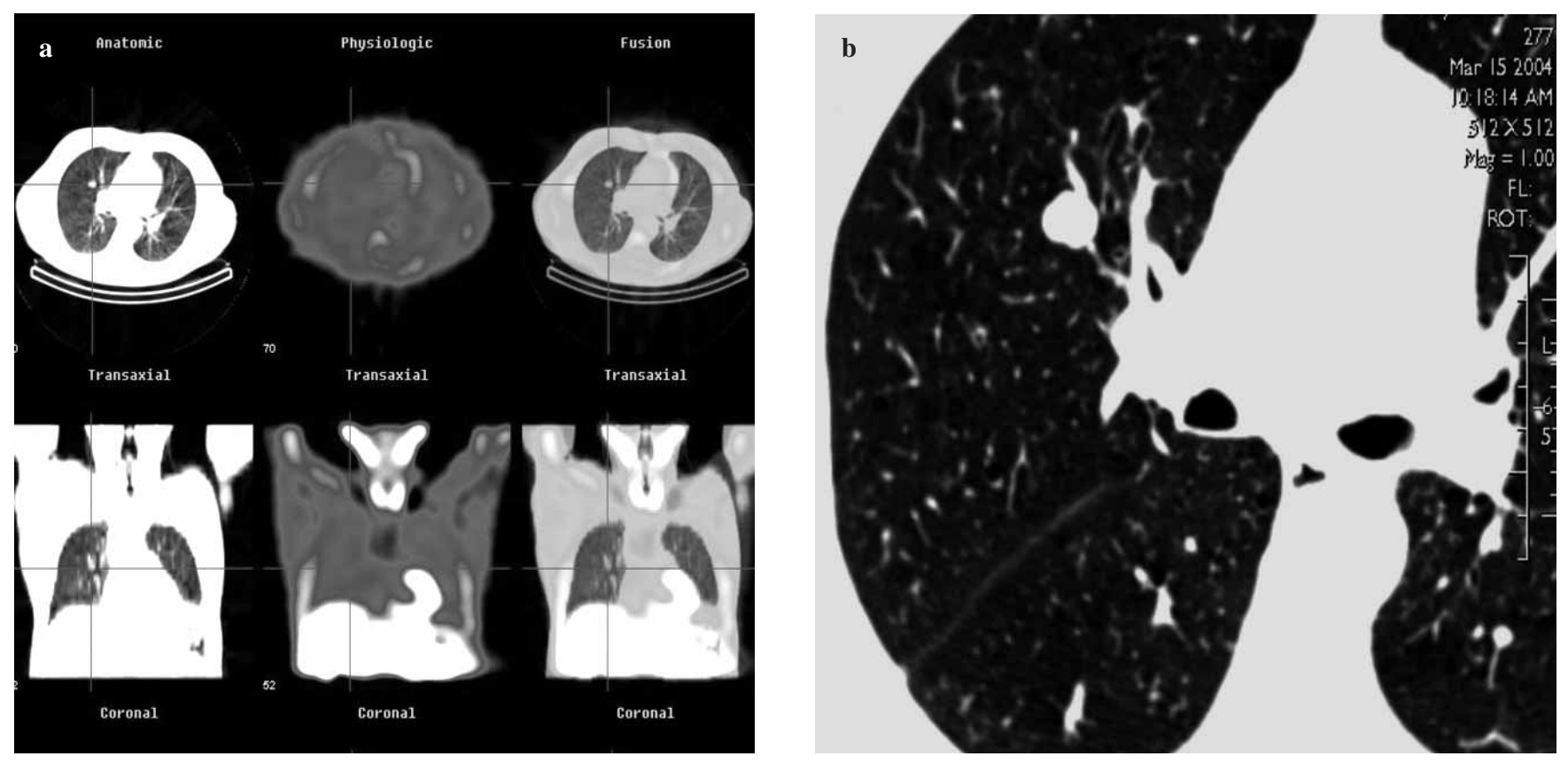

Fig. 2a Negative lung Tc-99m Sestamibi single photon emission computed tomography (SPECT) scan. b The CT image shows a lobulated nodule (hamartoma).

Fig. 2a SPECT polmonare con Tc-99m Sestamibi negativa. b L'immagine TC dimostra una nodularità a margini lobulati (amartoma).
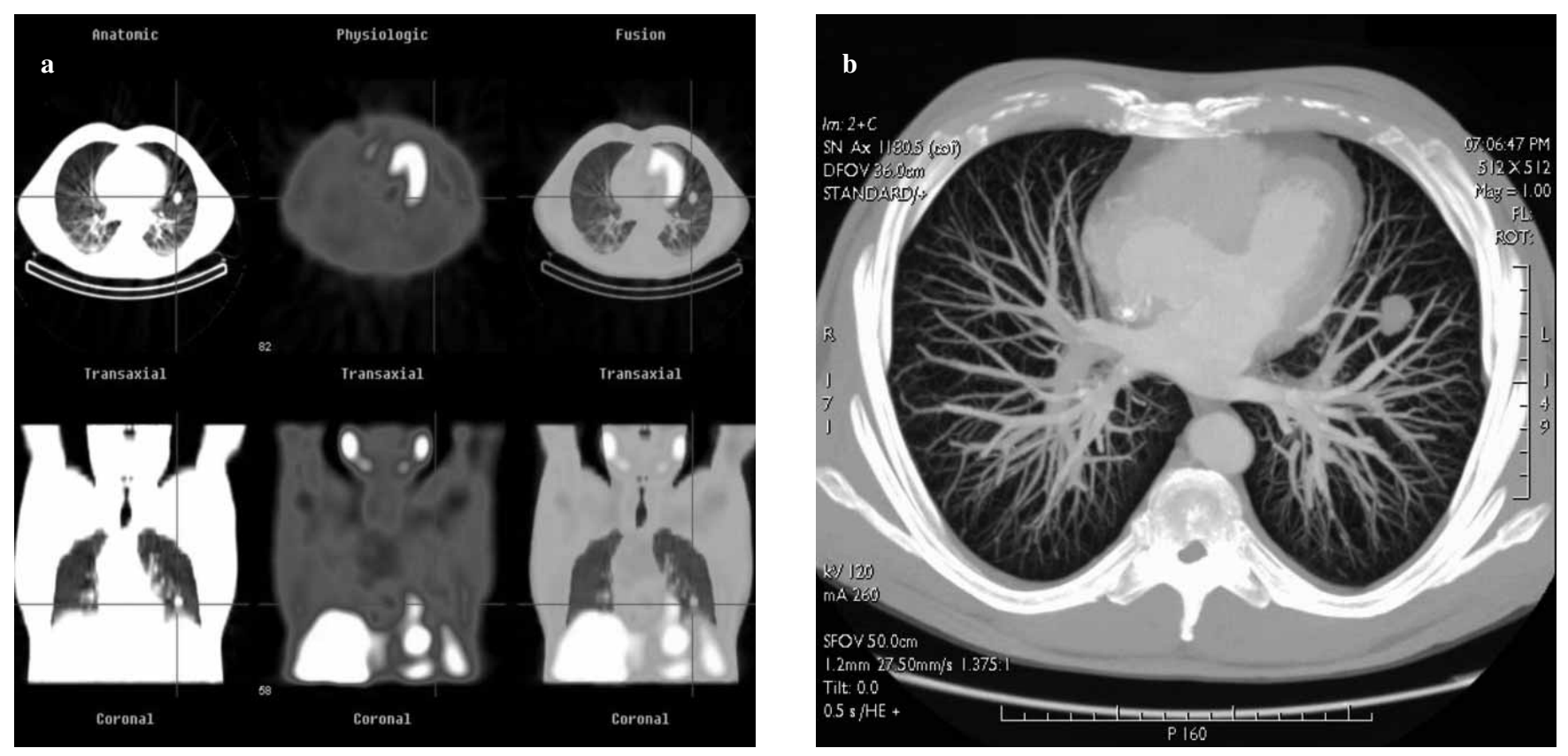

Fig. 3a A 63-year-old man with negative single photon emission computed tomography (SPECT) due to excessive focal uptake of the radiotracer. b Multiplanar reconstruction showing a juxtavascular nodule (hamartoma).

Fig. 3 a Uomo di 63 anni con esame SPECT negativo per iperaccumoli focali del radiotracciante. $\boldsymbol{b}$ Ricostruzione con algoritmo MIP che evidenzia un nodulo juxta-vascolare (amartoma). 
sion growth rate) criteria. In particular, the absence of growth over at least 2 years is a reliable indicator of benignity [16]. The use of 'doubling time', which for spherical le- lità (Se) del 90,9\%, una specificità (Sp) del 91,6\%, un'accuratezza diagnostica pari a $91,3 \%$ con un valore predittivo positivo (VPP) di 90,9\% ed un valore predittivo negativo (VPN)

Table 3 Nodule characterisation and follow-up

\begin{tabular}{|c|c|c|c|c|c|}
\hline Patients & SPECT & $\mathrm{CT}$ volume $\mathrm{t} 0, \mathrm{~cm}^{3}$ & $\begin{array}{l}\text { CT volume } \\
\text { at } 3-4 \text { months, } \mathrm{cm}^{3}\end{array}$ & Volume-doubling time & Histology \\
\hline 1 & Negative & 4.50 & 4.35 & Not applicable & Fibrous lesion \\
\hline 2 & Negative & 0.30 & 0.63 & $\sim 80$ days & Adenocarcinoma $^{a}$ \\
\hline 3 & Negative & 0.80 & 0.83 & $\sim 2,400$ days & Fibrous lesion \\
\hline 4 & Negative & 0.78 & 0.68 & Not applicable & Fibrous lesion \\
\hline 5 & Positive & 4.12 & & & Adenocarcinoma \\
\hline 6 & Negative & 4.03 & 3.98 & Not applicable & Fibrous lesion \\
\hline 7 & Positive & 0.73 & & & Adenocarcinoma \\
\hline 8 & Positive & 0.33 & & & Metastasis \\
\hline 9 & Positive & 1.04 & & & Adenocarcinoma \\
\hline 10 & Positive & 4.33 & & & Undifferentiated carcinoma \\
\hline 11 & Negative & 0.57 & 0.51 & Not applicable & Fibrous lesion \\
\hline 12 & Positive & 0.88 & & & GI Granuloma ${ }^{b}$ \\
\hline 13 & Positive & 1.63 & & & Squamous cell carcinoma \\
\hline 14 & Negative & 1.48 & 1.43 & Not applicable & Hamartoma \\
\hline 15 & Positive & 4.01 & & & Adenocarcinoma \\
\hline 16 & Positive & 1.60 & & & Adenocarcinoma \\
\hline 17 & Negative & 1.55 & 1.40 & Not applicable & Hamartoma \\
\hline 18 & Negative & 4.25 & 4.90 & $\sim 588$ days & Hamartoma \\
\hline 19 & Negative & 0.55 & 0.52 & Not applicable & Granuloma \\
\hline 20 & Positive & 0.81 & & & Adenocarcinoma \\
\hline 21 & Negative & 0.71 & 0.70 & Not applicable & Granuloma \\
\hline 21 & Positive & 0.44 & & & Adenocarcinoma \\
\hline 23 & Negative & 1.11 & 1.20 & $\sim 1,109$ days & Granuloma \\
\hline
\end{tabular}

${ }^{\mathrm{a}} \mathrm{False}$ negative; ${ }^{\mathrm{b}} \mathrm{False}$ positive

Tabella 3 Caratterizzazione dei noduli e follow-up

\begin{tabular}{|c|c|c|c|c|c|}
\hline Paziente & $S P E C T$ & Volume, TC t0, $\mathrm{cm}^{3}$ & $\begin{array}{l}\text { Volume, } \\
\text { TC } \Delta t \text { 3-4 mesi, } \mathrm{cm}^{3}\end{array}$ & $\begin{array}{l}\text { Tempo di raddoppio } \\
\text { volumetrico }\end{array}$ & Istologia \\
\hline 1 & Negativa & 4,50 & 4,35 & Non applicabile & Esito fibrotico \\
\hline 3 & Negativa & 0,80 & 0,83 & $\sim 2400 \mathrm{gg}$ & Esito fibrotico \\
\hline 4 & Negativa & 0,78 & 0,68 & Non applicabile & Esito fibrotico \\
\hline 5 & Positiva & 4,12 & & & Adenocarcinoma \\
\hline 8 & Positiva & 0,33 & & & Metastasi \\
\hline 9 & Positiva & 1,04 & & & Adenocarcinoma \\
\hline 10 & Positiva & 4,33 & & & $K$ indiff. \\
\hline 11 & Negativa & 0,57 & 0,51 & Non applicabile & Esito fibrotico \\
\hline 12 & Positiva & 0,88 & & & Granuloma gig. ${ }^{b}$ \\
\hline 17 & Negativa & 1,55 & 1,40 & Non applicabile & Amartoma \\
\hline 18 & Negativa & 4,25 & 4,90 & $\sim 588 \mathrm{gg}$ & Amartoma \\
\hline 19 & Negativa & 0,55 & 0,52 & Non applicabile & Granuloma sp. \\
\hline 20 & Positiva & 0,81 & & & Adenocarcinoma \\
\hline 21 & Negativa & 0,71 & 0,70 & Non applicabile & Granuloma \\
\hline 22 & Positiva & 0,44 & & & Adenocarcinoma \\
\hline 23 & Negativa & 1,11 & 1,20 & $\sim 1109 \mathrm{gg}$ & Granuloma \\
\hline
\end{tabular}


sions is defined as a $26 \%$ increase in diameter, relies on the observation that benign lesions double their volume in less than 30 days and more than 450 days. Extremely rapidly growing masses are probably inflammatory while slowgrowing masses fall under the category of so-called pseudodiseases, a term indicating diseases that never progress over a lifetime either because they do not increase in size and may in fact regress naturally (type I pseudodiseases) or be-
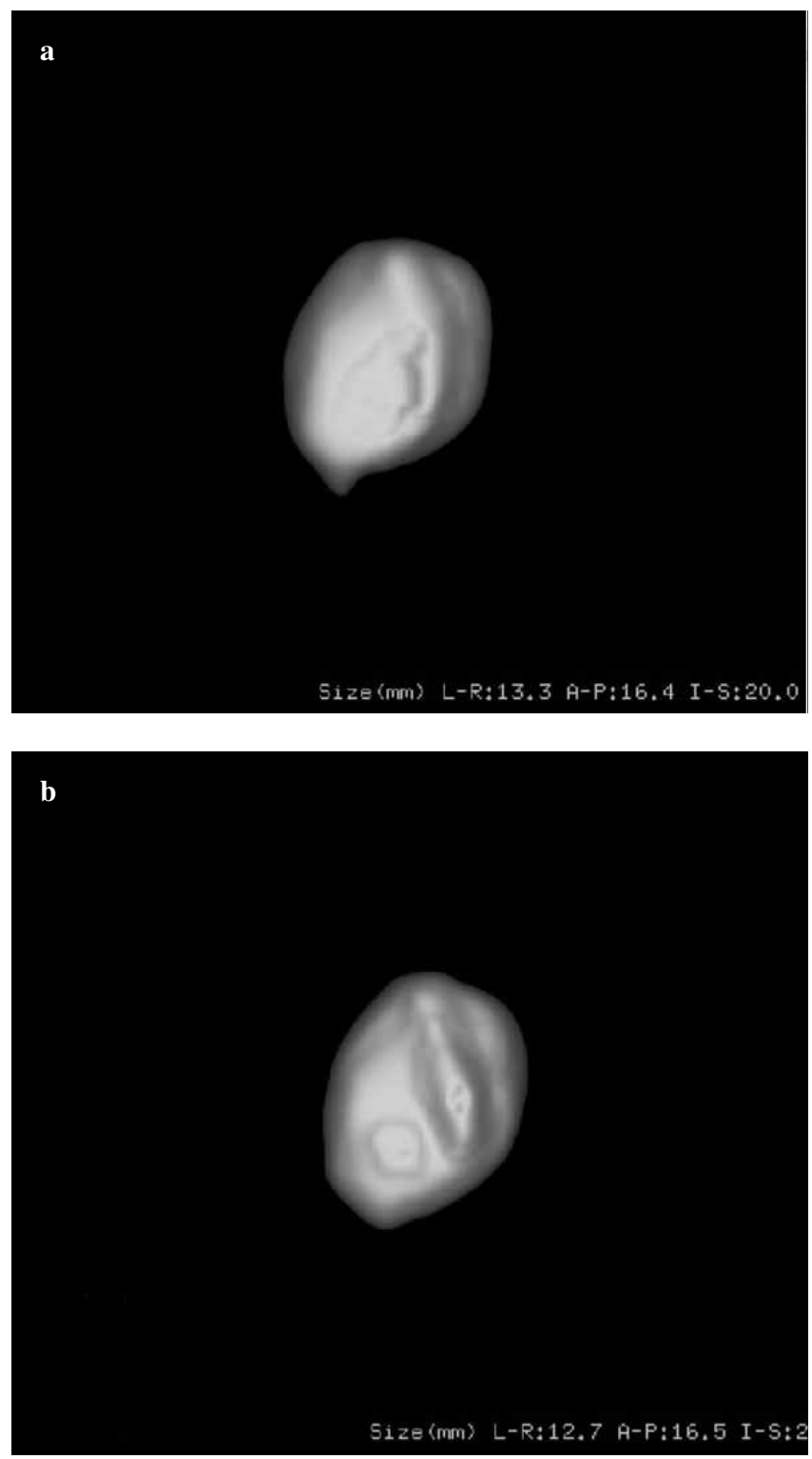

Fig. 4a,b Automatic three-dimensional segmentation [Advanced Lung Analysis software (ALA)] of the nodule and volume computation. a First examination in December 2003: a solitary pulmonary nodule with a volume of $1.488 \mathrm{~cm}^{3}$. b Second computed tomography (CT) examination in March 2004: nodule volume is $1.432 \mathrm{~cm}^{3}$. Growth rate: negative. Volume-doubling time: not applicable. Histological response: granuloma.

Fig. 4a,b Segmentazione automatica (ALA) del nodulo e calcolo volumetrico. a Prima identificazione a dicembre 2003: NPS con volume pari a $1.488 \mathrm{~cm}^{3}$. b Secondo esame TC a marzo 2004: il volume del NPS risulta pari a $1.432 \mathrm{~cm}^{3}$. Percentuale di crescita: negativa. Tempo di raddoppio del volume: non valutabile. Referto istologico: granuloma. pari a 91,6\% (Tabella 2). La consensuale acquisizione TC nella valutazione dell'esame scintigrafico è risultata sempre determinante nell'individuazione delle lesioni nodulari non captanti ed ha orientato verso una diagnosi differenziale nei casi di nodularità captanti localizzate in sede peri-epatica. La sola registrazione SPECT non adiuvata dall'immagine anatomica TC è risultata determinante in tutte le lesioni polmonari captanti a distribuzione diversa da quella peri-epatica. Lo studio TC, mediante valutazione volumetrica a 3-4 mesi, ha presentato una concordanza assoluta con il dato istologico (Tabella 3).

\section{Discussione}

Il convenzionale esame radiografico del torace, primo ed essenziale momento diagnostico per tutta la patologia polmonare, non si è dimostrato sufficientemente sensibile e specifico per assicurare una diagnosi precoce [10]. In passato, esami diagnostici basati sulla radiografia standard del torace e sull'esame citologico dell'escreato non hanno dato i risultati sperati nella riduzione della mortalità [11-13].

Negli ultimi anni si sta assistendo ad una profonda revisione del concetto di diagnosi precoce del cancro del polmone grazie agli incoraggianti risultati ottenuti con la tomografia computerizzata multistrato $[8,14,15]$, ed a tecniche complementari di medicina nucleare. Un NPS è definito come una opacità rotondeggiante intraparenchimale moderatamente ben delimitata con diametro massimo inferiore a 3 $\mathrm{cm}$ [1]. Nella differenziazione tra lesioni benigne e maligne in TC sono stati individuati criteri qualitativi, basati sulla morfologia del nodulo e quantitativi, rappresentati dalla densità, caratteristiche di enhancement dopo somministrazione di MdC organoiodato e dalla velocità di crescita della lesione nei casi sottoposti a controllo. In particolare l'assenza di crescita in un periodo di almeno 2 anni è un indicatore attendibile di benignità [16].

L'uso del "tempo di raddoppio", che per le lesioni sferiche è definito come un incremento del $26 \%$ del diametro, si basa sull'osservazione che le lesioni benigne raddoppiano il proprio volume in un tempo inferiore a $30 \mathrm{gg}$ e superiore a $450 \mathrm{gg}$. Infatti, le formazioni a rapidissimo accrescimento sono di probabile significato infiammatorio, mentre quelle a lenta crescita rientrano nel novero della così detta pseudomalattia ove con tale termine si intenda una malattia che non progredirà mai in senso negativo nel corso della vita, o perché non aumenta mai di dimensioni, potendo regredire naturalmente (pseudomalattia di tipo I) o perché progredisce cosi lentamente che non si svilupperanno mai $i$ sintomi nel corso della vita (pseudomalattia di tipo II) [17]. Un NPS che cresca ad una velocità tale che il suo tempo di raddoppio volumetrico previsto si collochi tra i 30 ed i $450 \mathrm{gg}$ deve essere sottoposto ad ulteriori accertamenti in quanto sospetto per malignità. L'utilizzo di programmi di segmentazione automatica (ALA) permette, con elevata accuratezza e ripetibilità, di calcolare le modificazioni volumetriche di NPS in uno stesso paziente valutato in tempi diversi e la significatività della crescita del nodulo [18] (Fig. 4). Il Tc-99m Sesta- 
cause they progress so slowly that patients never develop symptoms (type-II pseudodiseases) [17]. An SPN growing at such a rate that its volume-doubling time ranges from 30 to 450 days needs to be further investigated as it is suspicious for malignancy.

The use of automatic segmentation software (ALA) allows, with high accuracy and repeatability, the calculation of volume changes of the SPN in a patient assessed at different times and the significance of the nodule's growth [18] (Fig. 4). Tc-99m Sestamibi is a radiopharmaceutical usually employed in the study of myocardial perfusion, which has also shown to be effective in cancer diagnostics. The mechanism through which the uptake occurs in cancer cells is still uncertain, and some studies [19] have suggested a role for the negative potential of cell and mitochondrial membranes, passive diffusion, and the lipophilic nature of methoxyisobutyl isonitrile (MIBI). Tc-99m Sestamibi has therefore been used as an 'oncotropic' radiopharmaceutical in the study of various types of cancer, including lung cancer [20]. In agreement with the literature [21, 22], in all cases examined and subsequently subjected to histopathology, the use of SPECT with Tc-99m Sestamibi often provided support for radiological suspicion, but in selected highly suspicious cases, it prompted surgical excision rather than follow-up. The results suggest, consistent with the literature data $[21,22]$, that Sestamibi has a high specificity in the identification of malignant lung lesions.

Although our preliminary data are based on a small case series and require confirmation by larger studies, they suggest that SPECT-CT can detect true positive cases at an early stage. Therefore, the possible inclusion of scintigraphy in the characterisation of SPNs might lead to $\mathrm{CT}$ being reserved for the follow-up of scintigraphically negative lesions only. With regard to sensitivity, SPECT yielded one false negative result, in an 8-mm lesion (the smallest in our series).

However, even biological factors such as cancer aggressiveness and the possible presence of multidrug resistance may affect results. The mechanism for the uptake of Tc-99m Sestamibi - a lipophilic cation that is taken up by cancer cells - is related to regional blood flow and to the activity of mitochondria, where it is almost exclusively concentrated $[23,20]$. It has also been observed that cancer cells, characterised by chemoresistance, show a rapid washout of Tc-99m Sestamibi, which is linked to increased expression of certain membrane P-glycoproteins [24, 25].

The main benefit of SPECT-CT fusion imaging was, in our experience, the correct characterisation of perihepatic lesions affected by the normal uptake of the radiopharmaceutical by the liver. The clinical application of SPECT-CT to cancer diagnostics proved to be very useful in preliminary studies published in the literature [26]. Fusion of functional and anatomical images provides accurate localisation of scintigraphic findings, with significant improvement in diagnostic accuracy of the SPECT scans. Our work, although based on a limited number of patients, confirms preliminary results on the potential of this new technique in the differentiation of lung lesions with lipophilic cationic radiopharmaceuticals based on technetium [5]. The use of this radiotracer would also assist in cost-benefit decision making for pa- mibi è un radiofarmaco impiegato solitamente nello studio della perfusione miocardica, ma dimostratosi efficace nella diagnostica oncologica. Il meccanismo attraverso il quale avviene l'up-take nella cellula neoplastica è ancora incerto ed alcuni studi [19] suggeriscono il ruolo del potenziale negativo delle membrane cellulari e mitocondriali, della diffusione passiva, e della natura lipofilica del MIBI. In virtù di questo il Tc-99m Sestamibi è stato utilizzato come radiofarmaco "oncotropo" per la studio di diverse neoplasie, incluso il tumore polmonare [20]. Nell'ambito del nostro studio l'applicazione della SPECT-TC con Tc-99m Sestamibi, sulla base di precedenti in letteratura [21, 22], in tutti i casi da noi esaminati e successivamente sottoposti a diagnosi anatomo-patologica, ha fornito da un lato spesso un conforto al sospetto radiologico, dall'altro, in casi selezionati fortemente sospetti, ha fatto optare per l'escissione chirurgica piuttosto che per il follow-up. I risultati riportati suggeriscono, in accordo ai dati raccolti in letteratura [21, 22], un'alta specificità del Sestamibi nell'identificazione di lesioni polmonari maligne. I nostri dati preliminari, ottenuti in una casistica limitata, se confermati in studi successivi con l'inclusione di un numero maggiore di pazienti, suggeriscono che la SPECT-TC è in grado di identificare precocemente i casi veri positivi. Pertanto la possibile inclusione della scintigrafia nella caratterizzazione del NPS, potrebbe in futuro riservare il follow-up TC alle sole lesioni non captanti. Per quanto riguarda la sensibilità della SPECT-TC, va sottolineato che l'unico caso risultato falso negativo è stato osservato in una lesione di $8 \mathrm{~mm}$ (la più piccola dimensionalmente nella nostra casistica). Tuttavia, anche fattori biologici quali l'aggressività della neoplasia e la possibile presenza di una multidrug resistance possono essere influenti. Infatti il meccanismo di accumulo del Tc-99m Sestamibi, che è un catione lipofilico che viene captato dalle cellule neoplastiche, è in relazione al flusso ematico distrettuale ed alla attività mitocondriale, dove si concentra quasi esclusivamente [23, 20]. È stato poi osservato che nelle cellule neoplastiche, caratterizzate da chemioresistenza, si ha un rapido wash-out del Tc-99m Sestamibi, legato ad un'aumentata espressione di alcune P-glicoproteine di membrana [24, 25]. Il vantaggio più importante della fusione delle immagini SPECT-TC, nella nostra esperienza, si è avuto nella corretta caratterizzazione delle lesioni peri-epatiche, condizionate dal normale up-take del radiofarmaco da parte del fegato. L'impiego clinico della SPECT-TC in diagnostica oncologica si è rivelato molto utile nelle esperienze preliminari pubblicate in letteratura [26]. Infatti la fusione delle immagini funzionali con quelle anatomiche, permette una precisa localizzazione dei reperti scintigrafici, con un importante miglioramento dell'accuratezza diagnostica degli esami SPECT. Il nostro lavoro, anche se basato su un numero non elevato di pazienti, conferma le acquisizioni preliminari circa le potenzialità di questa nuova tecnica nella diagnostica differenziale delle lesioni polmonari con radiofarmaci cationici lipofilici tecneziati [5]. L'utilizzo di questo radiotracciante sarebbe inoltre di ausilio nell'ottica decisionale costo-beneficio, nei pazienti che, per la compromessa funzionalità cardio-polmonare, risulterebbero ad alto rischio per una toracotomia. Altro aspetto non trascurabile è la maggiore disponibilità sul ter- 
tients with impaired cardiopulmonary function and ineligible for thoracotomy. Another important aspect is that SPECT is more widely available and less expensive than positron emission tomography-fluorodeoxyglucose (PET-FDG), an examination that is sensitive and specific in diagnosis and staging of lung cancer $[27,28]$ but currently limited by its cost and poor availability. PET-FDG, however, has an accuracy of $90 \%$ and sensitivity and specificity of $91 \%$ and $68 \%$, respectively, in the differential diagnosis of SPNs [29].

\section{Conclusions}

In conclusion, the joint use of CT and Sestamibi SPECT in managing a patient with an SPN might represent another diagnostic tool in the differentiation of benign and malignant lung lesions in the future. ritorio delle apparecchiature ed il basso costo dell'esame, se confrontato con la PET-FDG, esame dimostratosi sensibile e specifico nella diagnosi e nella stadiazione delle neoplasie polmonari [27, 28], ma attualmente limitato dai costi e dalla scarsa reperibilità su territorio. La PET-FDG, comunque, dimostra nella diagnosi differenziale del NPS una accuratezza del $90 \%$, con una sensibilità ed una specificità del $91 \%$ e $68 \%$ rispettivamente [29].

\section{Conclusioni}

In conclusione, l'utilizzo consensuale della TC e della SPECT con Sestamibi nella gestione del paziente portatore di un NPS potrebbe rappresentare in futuro un ulteriore strumento diagnostico nell'iter differenziale tra lesioni polmonari benigne e maligne.

\section{References/Bibliografia}

1. Erasmus J, Connolly J, McAdams H et al (2000) Solitary pulmonary nodules: Part I - Morphologic evaluation for differentiation of benign and malignant lesions. Radiographics 20:43-58

2. Hassan IM, Sahweil A, Constantinides C et al (1989) Uptake of 99mTc hexakis 2-methoxyisobutylisonitrile in benign and malignant lesions in the lungs. Clin Nucl Med 14:333-340

3. Kao CH, Wang SJ, Lin WY et al (1993) Differentiation of single solid lesions in the lungs by means of single-photon emission tomography with technetium99m methoxyisobutylisonitrile. Eur J Nucl Med 20:249-254

4. Abdel-Dayem HM, Scott A, Macapinlac $\mathrm{H}$ et al (1994) Tracer imaging in lung cancer. Eur J Nucl Med 21:57-81

5. Schillaci O, Danieli R, Manni C, Simonetti G (2004) Is SPECT/TC with a hybrid camera useful to improve scintigraphic imaging interpretation? Nucl Med Comm 25:705-710

6. Schillaci O, Simonetti G (2004) Fusion imaging in nuclear medicine applications of dual-modality systems in oncology. Cancer Biother Radiopharm 19:1-10

7. Szabo E, Birrer MJ, Mulschine JL (1993) Early detection of lung cancer. Semin Oncol 20:374-382

8. Diederich S, Wormanns D, Semik M et al (2002) Screening for early lung cancer with low-dose spiral CT: prevalence in 817 asymptomatic smokers. Radiology 222:773-781
9. Bocher M, Balan A, Krausz Y et al (2000) Gamma camera-mounted anatomical X-ray tomography: technology, system characteristics and first images. Eur J Nucl Med 27:619-627

10. Parkin DM, Moss SM (2000) Lung cancer screening: improved survival but no reduction in deaths - the role of overdiagnosis. Cancer 89[Suppl 11]:236-276

11. Kubik AK, Zatloukal P, Boyle P et al (2001) A case-control study of lung cancer among Czech women. Lung Cancer 31:111-122

12. Sobue T (2000) A case-control study for evaluating lung cancer screening in Japan. Cancer 89[Suppl 11]:2392-2396

13. Black WC (2000) Overdiagnosis: an under-recognized cause of confusion and harm in cancer screening. J Natl Cancer Inst 92:1280-1282

14. Nawa T, Nakagawa $T$, Kusano $S$ et al (2002) Lung cancer screening using low dose spiral CT: results of baseline and 1year follow-up studies. Chest 122:15-20

15. Sone S, Li F, Yang ZG, Honda T et al (2001) Results of three-year mass screening programme for lung cancer using mobile low-dose spiral computed tomography scanner. Br J Cancer 84:25-32

16. Nathan MH, Collins VP, Adams RA (1962) Differentiation of benign and malignant pulmonary nodules by growth rate. Radiology 79:221-232
17. Obuchowski NA, Graham RJ, Baker ME, Powell KA (2001) Ten criteria for effective screening: their application to multislice CT screening for pulmonary and colorectal cancers. AJR Am J Roentgenol 212:1357-1362

18. Yankelevitz D, Gupta R, Zhao B et al (1999) Small pulmonary nodules: evaluation with repeat CT-preliminary experience. Radiology 212:561-566

19. Delmon-Moingeon LI, Piwnica-Worms D, Van den Abbeele AD (1990) Uptake of the cation hexakis (2-methoxyisobutylisonitrile)-technetium-99m by human carcinoma cell lines in vitro. Cancer Res 50:2198-2202

20. Maffioli L, Steens J, Pauweles E et al (1996) Applications of 99mTc-sestamibi in oncology. Tumori 82:12-21

21. Minai OA, Raja $S$, Metha AC et al (2000) Role of 99mTc MIBI in the evaluation of single pulmonary nodules: a preliminary report. Thorax 55:60-62

22. Nosotti M, Santambrogio L, Gasparini $\mathrm{M}$ et al (2002) Role of $99 \mathrm{mTc}$-hexakis2-methoxy-isobutylisonitrile in the diagnosis and staging of lung cancer. Chest 122:1361-1364

23. Chiu DA, Kronauge JF, Piwnica-Worms D (1990) Effect of mitochondrial and plasma membrane potentials on accumulation of hexakis (2-methoxyisobutylisonitrile) technetium (I) in cultured mouse fibroblast. J Nucl Med 31:1646-1653 
24. Sasaki M, Kuwabara Y, Ichiya Y et al (1999) Prediction of the chemosensitivity of lung cancer by $99 \mathrm{mTc}$-hexakismethoxyisobutylisonitrile SPECT. J Nucl Med 40:1778-1783

25. Sharma V (2004) Radiopharmaceuticals for assessment of multidrug resistance P-glycoprotein-mediated drug transport activity. Bioconjug Chem 15:1464-1474
26. Schillaci O (2005) Hybrid SPECT/CT: a new era for SPECT imaging? Eur J Nucl Med Mol Imaging 32:521-524

27. Lowe VJ, Naunheim KS (1998)

Positron emission tomography in lung

cancer. Ann Thorac Surg 65:1821-1829
28. Dewan NA, Gupta NC, Redepenning LS et al (1993) Diagnostic efficacy of PETFDG imaging in solitary pulmonary nodules. Chest 104:997

29. Gambhir S, Czernin J, Schwimmer J et al (2001) A Tabulated Summary of the FDG PET Literature. J Nucl Med 42:1-93 